\title{
MEF2A sequence variants and coronary artery disease: a change of heart?
}

\author{
David Altshuler1,2,3 and Joel N. Hirschhorn'1,3,4
}

\begin{abstract}
1Department of Genetics, Harvard Medical School, Boston, Massachusetts, USA. ²Department of Molecular Biology, Center for Human Genetic Research, and Diabetes Unit, Department of Medicine, Massachusetts General Hospital, Boston, Massachusetts, USA. 3Program in Medical and Population Genetics, Broad Institute of Harvard and Massachusetts Institute of Technology, Cambridge, Massachusetts, USA. ${ }^{4}$ Divisions of Genetics and Endocrinology, Children's Hospital, Boston, Massachusetts, USA.
\end{abstract}

\begin{abstract}
Rare mutations in $M E F 2 A$ have been proposed as a cause of coronary artery disease (CAD) and myocardial infarction (MI). In this issue of the JCI, Pennacchio and colleagues report sequencing MEF2A in 300 patients with premature CAD and in controls. Only 1 CAD patient was found to carry a missense mutation not found in controls. The specific 21-bp deletion in $M E F 2 A$ previously proposed as causal for CAD and/or MI was observed in unaffected individuals and did not segregate with CAD in families (see the related article beginning on page 1016). These results do not support the hypothesis that mutations in MEF2A are a cause of CAD and/or MI but do illustrate general principles regarding the difficulty of connecting genetic variation to common diseases.
\end{abstract}

Known risk factors explain only a small fraction of interindividual risk of coronary artery disease (CAD). An important clue to understanding the etiology of CAD is its substantial heritability, which demonstrates that variation in DNA sequence influences risk (1). It was recently proposed that mutations in $M E F 2 A$, which encodes a member of the myocyte enhancer factor-2 (MEF2) family of transcription factors, are a cause of CAD and myocardial infarction (MI) (2), a finding that, if true, would provide an important and previously unsuspected insight into the pathogenesis of this clinically important disease. In this issue of the JCI, however, Pennacchio and colleagues report that rare missense changes in $M E F 2 A$ are not observed at a substantial frequency in patients with early-onset CAD (3). More critically, they discovered that a specific 21-bp deletion in $M E F 2 A$ - originally claimed to be the causal mutation in the index family - is also present in unaffected individuals, where it does not cosegregate with CAD. How can we reconcile these results; what do they tell us about the proposed role of MEF2A in CAD; and what general principles do they highlight

Nonstandard abbreviations used: CAD, coronary artery disease; LOD, logarithm of the odds; MEF2, myocyte enhancer factor- 2 ; MI, myocardial infarction.

Conflict of interest: The authors have declared that no conflict of interest exists.

Citation for this article: J. Clin. Invest. 115:831-833 (2005). doi:10.1172/JCI200524715. about the challenges in reliably implicating genetic variants in human disease?

The study in this issue of the JCI was prompted by an earlier article published in Science (2) that described a large family with 21 members, 13 of whom were affected with a composite endpoint of CAD and/or MI: patients in whom stenosis has been detected upon catheterization or who have undergone a revascularization procedure or experienced past MI. Because 13 of 21 family members were affected, the authors hypothesized that in this family, CAD and/or MI was an autosomal-dominant, single-gene disorder. When the data were analyzed based on this assumption, significant evidence was obtained for linkage to chromosome 15 q26 (logarithm of the odds [LOD] score, 4.19), implicating a region that at a minimum contains 93 genes.

The authors of the earlier study analyzed only 1 of these 93 positional candidate genes, MEF2A, because it is expressed in the vasculature of embryonic mice (2). A 21-bp in-frame deletion was identified in one of the affected individuals from the family. The 21-bp deletion also segregated with disease in this family; however, this variant in $M E F 2 A$ was identified precisely because it resided in a large region that is tightly linked to CAD and/or MI in this family, so its cosegregation with disease status is expected and provides little new information. In this initial report, the 21-bp deletion was absent in 119 unrelated individuals with normal angiograms, and was found to alter the ability of the MEF2A protein to activate transcription in vitro.

The same group published a second article (4) in which they screened the MEF2A gene in 207 individuals with CAD and 191 controls. They found 3 different single nucleotide protein polymorphisms in a total of 4 CAD patients and none in controls. Proteins carrying these changes altered MEF2A transactivation activity in vitro. Based on these data, the authors concluded that each of these variants of MEF2A is a cause of $\mathrm{CAD}$ and/or MI and estimated from these data that approximately $2 \%$ of people with CAD carry such mutations.

Pennacchio and colleagues (3) now report the resequencing of the MEF2A coding region in 300 patients with premature CAD and in a control group of elderly unaffected individuals. Of the MEF2A missense variants identified in CAD patients, only a single variant was not also present in controls. This missense variant was located in an amino acid that is not conserved across species, which suggests that this amino acid is not crucial for $M E F 2 A$ function, although this was not evaluated experimentally.

More importantly, the 21-bp deletion that Wang et al. had claimed to be the causal mutation (2) was observed in a clinically unaffected control subject rather than in a patient with CAD (3). In the family of this control, the 21-bp deletion was found in 3 more elderly people without $\mathrm{CAD}$, and was absent from 2 patients who did have CAD. An additional 1,500 people without clinically apparent CAD were screened for the 21-bp deletion, which was identified in 2 families. None of the individuals carrying the 21-bp deletion in MEF2A had clinically apparent CAD or other cardiovascular diseases.

First, the 21-bp deletion in MEF2A initially claimed to be a causal mutation by Wang et al. (2) appears instead to be a rare polymorphism that does not consistently 
segregate with CAD and/or MI in families. Six subjects in 3 families were found who carried the 21-bp deletion and had other risk factors for CAD (age, dyslipidemia, hypertension, diabetes, and a history of smoking). None of these individuals showed evidence of CAD.

It is difficult to create a model in which the 21-bp deletion is sufficient to cause CAD and/or MI in one family and yet shows no association to disease in other families. The only possibility is that there could be a genetic or environmental modifier that is absolutely required for its effect. Because the original evidence of linkage rested heavily on the assumption of strict monogenic inheritance, however, and since relaxing this assumption would likely make the evidence for linkage much less significant, invoking an unspecified modifier locus may actually weaken the case for $M E F 2 A$ rather than strengthen it.

Second, the frequency of missense changes in CAD and controls does not provide an alternative argument in support of a causal role for $M E F 2 A$ variation in CAD and/or MI. The prevalence of rare, nonsynonymous changes in MEF2A identified in patients with CAD but not in healthy controls is very low, approximately $1 \%$ in the combined populations of studies published to date. The current estimate of the difference in the prevalence of nonsynonymous change between CAD and control patients ( 5 of 500 versus 0 of 500, respectively) does not achieve nominal significance $(P>0.2)$, much less the more stringent levels of significance required to overcome the relatively low prior probability that a given candidate gene is causal (ref. 5; see also refs. 6-8).

Finally, although results of functional studies of MEF2A variants have suggested alterations of function in vitro, these do not prove a role for MEF2A in CAD and/or MI. Because these in vitro assays have no documented relationship to $\mathrm{CAD}$ and/or $\mathrm{MI}$ in vivo, they are of limited value in assessing the likelihood of an etiological role for $M E F 2 A$ mutations in CAD and/or MI.

If the available evidence is not adequate to conclude that mutations in MEF2A play a causal role in CAD and/or MI, what general principles can we take away? First, evidence from more than one mutation in a single family is required when making a claim of causality. Because a single observation of a potentially functional variant (such as an in-frame deletion) is not an unusual event, multiplicity is required. Multiple different mutations, multiple independent observations of the same mutations, and/or multiple compelling lines of evidence are required to prove the link between variation in a gene and disease. Together these multiple variants should satisfy one or more of the following criteria. (a) Multiple different mutations exist, each of which is evidently functional and cosegregates with disease in human patients. (b) Enrichment of a particular allele in disease cases as compared with controls, with enough observations to establish statistical significance. (c) Enrichment in disease cases of different rare mutations, where the frequency of such mutations is ascertained with equal vigor in controls. The challenge here is deciding which of the observed changes should be lumped into the "causal" category in the disease cases as compared with the controls (7). Unless functional studies are performed, and unless the available assays bear a validated relationship to the disease in vivo, it is difficult to know which observed changes might be causal and which are clinically neutral variants. (d) Observation of a de novo mutation that is present in an affected individual (but not in his or her biological parents), which is extraordinarily rare given the low spontaneous mutation rate in humans. (e) Compelling effects of a human mutation in a model system, such as an in vivo mouse model with recapitulation of the human disease phenotype.

Because MEF2A was only one of approximately 93 candidate genes at $15 \mathrm{q} 26$, it is of course possible that another gene in the region may yet be found to play a role in CAD and/or MI. It is important to note, however, that while the initial LOD score above 4 is considered significant for a genome-wide linkage study, this result is only valid under the author's assumption that, in a family with 13 of 21 individuals affected, CAD and/or MI is segregating as a Mendelian, autosomal-dominant trait (2). In a common disease such as CAD, it is also possible that a large family could contain many affected individuals by chance alone, thus giving an appearance consistent with single-gene inheritance.

Detailed characterization of patient phenotype can be extremely important in such studies. The hypothesis of monogenic inheritance in a family is more compelling if the affected members share an unusual phenotype, for example, early onset of diabetes in maturity-onset diabetes of the young (9) or a syndromic form of disease as is the case in many disorders of blood pressure and electrolyte metabolism (10). No such distinctive phenotype was consistently evident in the CAD and/or MI family originally described (2), in which many of the affected individuals had a typical age of onset and clinical presentation of CAD. Under other models of inheritance, the linkage to $15 \mathrm{q} 26$ would likely not be as significant. Similarly, given other definitions of affected status (stenosis detected upon catheterization, a previous revascularization procedure, or past MI considered individually, rather than the composite of all 3), the linkage results would be different. Since parametric linkage analysis (in which a specific model of inheritance is postulated) can be powerfully influenced by the affectation status of even 1 or 2 individuals, more intensive analysis of unaffected family members can be important to rule out disease that has not yet manifested clinically.

Given the effort required for comprehensive study of the positional candidate genes at 15 q26, it would be valuable to observe more families with linkage to this region, applying a single definition of affectation status, before embarking on a large effort to track down other genes in this region. It is worth noting that multiple other studies have searched for linkage to premature CAD and/or MI, and have not highlighted linkage to this locus (1, 11-15), including a large study done by the authors of the original article on $M E F 2 A$ in Science (11).

In conclusion, $M E F 2 A$ may play an important role in cardiovascular biology, and rare variants in MEF2A may influence its activity as a transcription factor in vitro, but the genetic evidence available to date does not demonstrate that these mutations play a causal role in CAD and/or MI in humans. While further scrutiny of $M E F 2 A$ may help sort out its role, these studies (2-4) are also valuable in reminding us that replication and multiplicity in human genetic research are critically important; that linkage studies implicate a region, not a single gene; that there are limitations in extrapolating from a functional effect in vitro to a medical consequence in vivo; and that defining a mode of inheritance and proper definition of phenotype for common diseases is a challenge. As advances in technology make it easier to collect such data (but no easier to interpret them!), these principles will be increasingly important in the years to come. 


\section{Acknowledgments}

The authors would like to thank Richard Lifton and Christine Seidman for their advice and contributions to the text.

Address correspondence to: David Altshuler, Department of Molecular Biology, Massachusetts General Hospital, Boston, Massachusetts 02114, USA. Phone: (617) 726-5940; Fax: (617) 726-5937; E-mail: altshuler@molbio.mgh.harvard.edu. Or to: Joel N. Hirschhorn, Enders 561, Children's Hospital, 300 Longwood Avenue, Boston, Massachusetts 02115, USA. Phone: (617) 919-2129; Fax: (617) 730-0253; E-mail: joelh@broad.mit.edu.

1. Lusis, A.J., Mar, R., and Pajukanta, P. 2004. Genetics of atherosclerosis. Annu. Rev. Genomics Hum. Genet. 5:189-218.

2. Wang, L., Fan, C., Topol, S.E., Topol, E.J., and Wang,
Q. 2003. Mutation of MEF2A in an inherited disorder with features of coronary artery disease. Science. 302:1578-1581.

3. Weng, L., et al. 2005. Lack of MEF2A mutations in coronary artery disease. J. Clin. Invest. 115:1016-1020. doi:10.1172/JCI200524186.

4. Bhagavatula, M.R., et al. 2004. Transcription factor MEF2A mutations in patients with coronary artery disease. Hum. Mol. Genet. 13:3181-3188.

5. Wacholder, S., Chanock, S., Garcia-Closas, M., El Ghormli, L., and Rothman, N. 2004. Assessing the probability that a positive report is false: an approach for molecular epidemiology studies. J. Natl. Cancer Inst. 96:434-442.

6. Lohmueller, K., Pearce, C.L., Pike, M., Lander, E.S., and Hirschhorn, J.N. 2003. Meta-analysis of genetic association studies supports a contribution of common variants to susceptibility to common disease. Nat. Genet. 33:177-182.

7. Hirschhorn, J.N., and Altshuler, D. 2002. Once and again-issues surrounding replication in genetic association studies. J. Clin. Endocrinol. Metab. 87:4438-4441.

8. Dahlman, I., et al. 2002. Parameters for reliable results in genetic association studies in common disease. Nat. Genet. 30:149-150.
9. Florez, J.C., Hirschhorn, J., and Altshuler, D. 2003. The inherited basis of diabetes mellitus: implications for the genetic analysis of complex traits. Annu. Rev. Genomics Hum. Genet. 4:257-291.

10. Lifton, R.P., Gharavi, A.G., and Geller, D.S. 2001. Molecular mechanisms of human hypertension. Cell. 104:545-556.

11. Wang, Q., et al. 2004. Premature myocardial infarction novel susceptibility locus on chromosome 1P34-36 identified by genomewide linkage analysis. Am. J. Hum. Genet. 74:262-271.

12. Pajukanta, P., et al. 2000. Two loci on chromosomes 2 and $X$ for premature coronary heart disease identified in early- and late-settlement populations of Finland. Am. J. Hum. Genet. 67:1481-1493.

13. Hauser, E.R., et al. 2004. A genomewide scan for earlyonset coronary artery disease in 438 families: the GENECARD Study. Am. J. Hum. Genet. 75:436-447.

14. Francke, S., et al. 2001. A genome-wide scan for coronary heart disease suggests in Indo-Mauritians a susceptibility locus on chromosome 16p13 and replicates linkage with the metabolic syndrome on 3q27. Hum. Mol. Genet. 10:2751-2765.

15. Broeckel, U., et al. 2002. A comprehensive linkage analysis for myocardial infarction and its related risk factors. Nat. Genet. 30:210-214.

\title{
The fickle finger of fate
}

\author{
Luis de la Fuente and Jill A. Helms
}

Department of Surgery, Stanford University, Stanford, California, USA.

In this issue of the JCI, Niedermaier and colleagues demonstrate that a chromosomal inversion in mice results in dysregulation of Sonic hedgehog (Shh), such that $S h b$ is ectopically expressed in a skeletogenic domain typically occupied by Indian hedgehog (Ihh) (see the related article beginning on page 900). This molecular reversal eliminates phalangeal joint spaces, and consequently, Short digits $(D s h)$ heterozygotes $(D s h /+)$ have brachydactyly (shortened digits). I $b h$ is normally downregulated in regions that will become the joint space, but in $D s h /+$ mice, Shh bypasses this regulatory control and persists; accordingly, cells maintain their chondrogenic fate and the developed digits are shorter than normal. The significance of these data extends far beyond the field of skeletal biology: they hint at the very real possibility that the endogenous $S h b$ regulatory region contains a repressor designed to segregate the activity of Shh from Ihh. The existence of such a repressor provides a window into the distant past, revealing that $S h h$ and Ihh must once have shared responsibilities in establishing tissue boundaries and orchestrating vertebrate tissue morphogenesis.

Ancient physicians viewed the skeleton as "the foundation of the rest of the parts of the body and all the members rest upon them and are supported, as proceeding from a primary base" (1). Defects in this structural foundation also serve as portholes through which the process of

Nonstandard abbreviations used: Bmp, bone morphogenic protein; Dsh, Short digits; Dsh $/+$, Dsh heterozygote; Ihh, Indian hedgehog; Shh, Sonic hedgehog.

Conflict of interest: The authors have declared that no conflict of interest exists.

Citation for this article: J. Clin. Invest. 115:833-836 (2005). doi:10.1172/JCI200524840. fetal skeletogenesis can be analyzed. For example, studies of genetic perturbations that result in distal limb truncations have shown that the morphogen Sonic hedgehog (Shh) establishes precisely where skeletogenic condensations will form in the tips of the hand- and footplates $(2,3)$. This spatial patterning information is further refined by bone morphogenetic protein (Bmp) signaling, as shown by the fact that disruptions in the Bmp signaling pathway lead to fusions, or syndactyly, of the digits (4). Once the spatial pattern of the skeletogenic condensations is achieved, a closely related cousin, Indian hedgehog (Ihh), takes over and plays an instrumental role in segregating inner chondrogenic cells from the flattened, elongated perichondrial cells at the periphery (5). Ihh secreted from chondrocytes stimulates the differentiation of perichondrial cells into osteoblasts (5-7), mesodermal cells that give rise to bone.

Another critical feature of limb skeletogenesis is the creation of the articulations, or joint spaces, between the skeletal elements. In the fingers, joint spaces are created when a single, larger skeletogenic condensation cleaves into 2 or 3 smaller segments, each of which will give rise to a phalange (8). Wnt14 is critical in determining where a joint space will form (9), but precisely how the cleavage event is controlled remains uncertain. One thing is clear, however: when a separation fails to take place, the phenotypic consequence is brachydactyly (shortened digits). Thus, while we have a fairly complete picture of the range of skeletal malformations that can occur, how these disruptions are related to one another and to the basic program of skeletogenesis remains unknown.

\section{Reading the bones}

In this issue of the JCI, Niedermaier et al. provide new insights into the process of 